\title{
Avaliação e comparação de características de amortecimento de sinais gerados de diferentes violões
}

\author{
Evaluation and comparison of damping characteristics of signals \\ generated guitars different
}

\author{
Paulo Sérgio Teixeira ${ }^{1}$ \\ Alexandre José da Silva ${ }^{2}$ \\ José Flávio Silveira Feiteira ${ }^{3}$
}

1 Mestrando em Engenharia Metalúrgica e Materiais - UFF.
2 Doutor em Engenharia Mecânica - UFF.
3 Doutorado em Engenharia Mecânica - UFF.

\section{Resumo}

As taxas de amortecimento dos diferentes modos de vibração de um violão dependem de uma série de fatores. Entre esses, as propriedades do material das cordas. Há também a dissipação de energia também através do ar, onde as cordas ao serem excitadas sofrem resistência ao movimento devido a viscosidade do ar, e isso faz com que haja mais amortecimento. A taxa na qual a energia é perdida na irradiação do som depende de como os diferentes modos de corpo são excitados, e isso pode variar significativamente entre diferentes instrumentos. Essa taxa pode ser calculada através do decremento logarítmico, que é consequência de um simples impulso provocado no sistema (em vibração livre) e é obtido através da razão entre duas amplitudes sucessivas do sinal. 0 termo decremento logarítmico refere-se à taxa de redução logarítmica, relacionada com a redução do movimento após o impulso, pois a energia é transferida para outras partes do sistema ou é absorvida pelo próprio elemento. Neste trabalho foram calculadas as taxas de amortecimento da frequência fundamental de cada nota musical estudada. E se percebeu que as frequências entre 150 e 300 Hertz apresentam a melhor diferenciação entre os instrumentos.

\section{Palavras-chave}

Taxas de amortecimento; dissipação de energia; irradiação do som; decremento logarítmico.

\begin{abstract}
Damping rates of the different modes of vibration of a guitar depend on a number of factors. Among these, the material properties of the strings. There is also the power dissipation also through the air, where the strings to be excited suffer resistance to movement due to viscosity of the air, and this means that there is more cushioning. The rate at which energy is lost in the sound of the irradiation depends on how the different body modes are excited, and this can vary significantly between different instruments. This rate can be calculated by the logarithmic decrement, which is a result of a simple impulse caused the system (in free vibration) is obtained by the ratio of two successive amplitudes of the signal. The term logarithmic decrement refers to the logarithmic reduction rate relates to the reduction of movement after the boost, as the energy is transferred to other parts of the system or is absorbed by the element itself. In this work were calculated damping rates of the fundamental frequency of each studied musical note. And it was realized that the frequencies between 150 and 300 Hertz have better separation between the instruments.
\end{abstract}

\section{Keywords}

Damping rates; power dissipation; sound irradiation; logarithmic decrement.

\section{Como você deve citar?}

TEIXEIRA, Paulo Sérgio; SILVA, Alexandre José da; FEITEIRA, José Flávio Silveira. Avaliação e comparação de características de amortecimento de sinais gerados de diferentes violões. Cadernos UniFOA, Volta Redonda, n. 26, p. 17-30, dez. 2014. 


\section{INTRODUÇÃO}

Um desafio que merece intensa investigação está em entender corretamente e modelar os parâmetros que envolvem a construção de instrumentos musicais. As madeiras usadas em instrumentos musicais de boa qualidade como o violão ou o violino, possuem algumas características próprias responsáveis pela boa acústica de tais instrumentos, segundo informações de um dos principais luthiers, fabricante de violão, a lutheria faz uso de técnicas tradicionais que usam os sentidos humanos, como audição, por exemplo, para classificar madeiras para instrumentos. Uma das técnicas consiste em promover um leve impacto sobre a madeira e levá-la próxima ao ouvido e com a experiência do luthier, ele sabe, através do som emitido, se a madeira é boa ou não para o instrumento.

Um problema na construção desses instrumentos é que as madeiras consideradas de boa qualidade são madeiras nobres, muitas vezes escassas na natureza. Com o objetivo de possibilitar a substituição desses materiais às vezes já escassos na natureza ou de elevado custo, é necessário encontrar parâmetros quantitativos objetivos que permitam a avaliação de outras madeiras que tenham características semelhantes às tradicionalmente usadas na lutheria.

Através da análise de sinais acústicos de violões, objetiva-se desenvolver uma técnica de avaliação que possa auxiliar na análise da qualidade de diferentes instrumentos. 0 presente trabalho faz parte de um esforço para se identificar parâmetros mensuráveis nos sinais acústicos de violões e instrumentos congêneres e que possam estar ligados a qualidade dos materiais e madeiras e técnicas utilizadas na sua construção. $O$ presente trabalho concentra-se na análise dos sinais acústicos emitidos por violões que foram analisados no domínio de frequência e do tempo, de forma a permitir a determinação de parâmetros relacionados ao amortecimento das vibrações no instrumento.

Para tanto, aplicam-se de técnicas como a Transformada Fourier, bem como a filtragem de sinais por banda com o objetivo maior de estudar as características de amortecimento dos sinais.

\subsection{0 som no violão}

A produção de som no violão acontece quando a corda se deforma elasticamente. A corda vibra e excita o cavalete, com esforços transversais e de torsão deforma o tampo do instrumento. 0 tampo então vibra, excitando também o ar no interior e no exterior adjacente ao instrumento, produzindo um som audível. $O$ instrumento pode então ser entendido como um transdutor que transforma a energia mecânica cedida às cordas em energia acústica do ar. Como a área de contato entre as cordas e o ar é muito pequena, o instrumento e as características que ele empresta ao sinal acústíco produzido determinam de forma decisiva a qualidade do som produzido, em que pesam as características das vibrações das próprias. Em todas essas etapas, ocorre uma dissipação de energia ou amortecimento. No presente trabalho, procuramos enfocar o amortecimento causado pelo instrumento.

\subsection{Amortecimento dos sinais acústicos no violão}

Segundo Wright (1996) as taxas de amortecimento dos diferentes modos de vibração de um instrumento, enquanto um sistema vibracional composto de múltiplos materias e de forma complexa, dependem de uma série de fatores, inclusive das propriedades do material das cordas. Em cada parte do instrumento, a energia inicialmente cedida á deformação das cordas é irradiada na forma de som, mas também rapidamente dissipada, transformando se, em última análise, em energia interna dos materiais. Há também a dissipação de energia também através do ar, onde as cordas ao serem excitadas sofrem resistência ao movimento devido a viscosidade do ar, e isso faz com haja mais amortecimento. Segundo Wright (1996) 0 amortecimento imposto pelo ar é mais significativo em frequências mais altas 
onde a corda vibra mais rapidamente e tem de trabalhar mais para superar o atrito devido ao ar. Uma pequena quantidade de energia também pode ser perdida através do braço do instrumento, mas que provavelmente contribui pouco para o som irradiado. A energia perdida pela corda para o corpo vai ser a influência dominante sobre $o$ amortecimento das vibrações da corda para a maioria das freqüências. A taxa na qual a energia é perdida na irradiação do som depende de como os diferentes modos de corpo são excitados, e isso pode variar significativamente entre diferentes instrumentos.

Segundo Jannson (1983), o tom do violão tem dois componentes - as parciais das cordas e o som do corpo. 0 som do corpo decai rapidamente após excitado, mas tem um papel importante no tom. 0 músico pode mudar o espectro parcial do tom de forma previsível, variando a posição, forma e direção de tocar a corda. Mais perto do cavalete, excitam componentes mais fortes de alta frequência. 0 arranque em paralelo com o tampo superior dá um tom fraco, mas com grande duração.

Propriedades fundamentais do tom do violão, como os níveis iniciais de amplitude e quedas de nível inicial são definidas pelas propriedades do corpo do violão. Um elevado nível inicial significa um decaimento rápido inicial, ou seja, o nível inicial é proporcional ao nível de decaimento inicial. E a duração tende a ser mais prolongada quando os niveis iniciais são menores, mas isso depende dos fatores anteriormente relacionados.

\subsection{Amortecimento de Sinais}

Segundo Cossolino e Pereira (2010), 0 amortecimento em materiais elásticos, ou atrito interno, é uma das propriedades mais sensíveis de materiais e estruturas, tanto em escala macro quanto microscópica. É o fenômeno pelo qual a energia mecânica de um sistema é dissipada (principalmente pela geração de calor e/ou energia). 0 amortecimento determina a amplitude de vibração na ressonância e o tempo de persistência da vibração depois de cessada a excitação.

Segundo Cossolino e Pereira (2010), 0 amortecimento de um sistema ou material pode ser classificado de três formas principais: interno, estrutural e fluídico. 0 interno está associado aos defeitos na microestrutura, granularidade e impurezas do material e a efeitos termoelásticos causados por gradientes locais de temperatura. Já o estrutural está associado a perdas de energia por atrito em juntas, parafusos e articulações semi-rígidas. Por último, o fluídico ocorre por resistência ao arraste em meio fluídico, por exemplo, a conversão de energia cinética de um pêndulo em energia térmica para o ar.

Existem diversos métodos para determinação do amortecimento, os quais podem ser obtidos basicamente por dois caminhos: mediante a duração da resposta do sistema a uma excitação transitória (exemplo: método do decremento logarítmico) e em função da resposta do sistema em função da frequência (exemplo: método da largura de meia banda de potência). 0 método do decremento logarítmico calcula o amortecimento a partir da atenuação da resposta acústica do material ou estrutura após uma excitação por impulso. 0 método da largura de meia banda de potência calcula o amortecimento através da análise da frequência do sinal oriundo da vibração, a partir da relação entre a largura de banda e a frequência central de uma ressonância. Ambos os métodos consideram um modelo para os cálculos, normalmente o modelo de amortecimento viscoelástico. A escolha do método depende principalmente da faixa do amortecimento e da freqüência de vibração.

A importância de se calcular o amortecimento está no fato de que este parâmetro pode indicar como a energia de vibração é absorvida pelo meio material onde se propaga. 


\subsubsection{Amortecimento Viscoelástico}

No modelo viscoelástico considera-se que a natureza do amortecimento é viscosa e a força de atrito como sendo proporcional à velocidade, representando uma oposição ao movimento, sendo descrita pela equação:

$$
F=c \cdot \dot{x} ; c>0
$$

Fonte: Adaptada de (COSSOLINO; PEREIRA, 2010).

onde c é uma constante de proporcionalidade e $x$ a velocidade de deslocamento de uma massa em relação um ponto fixo. Como exemplo de um sistema com amortecimento, pode-se imaginar um pistão dentro de um cilindro preenchido com um líquido, considerando o sistema como massa-mola-amortecedor com um grau de liberdade. Sendo $m$ a massa, $k$ a constante elástica da mola e $c o$ coeficiente de amortecimento viscoso, pode-se representar este sistema pela seguinte equação:

$$
m \ddot{x}+c \dot{x}+k x=0
$$

Fonte: Adaptada de (COSSOLINO; PEREIRA, 2010).

Dividindo-se a equação por $m$, tem-se:

$$
\ddot{x}+\left(\frac{c}{m}\right) \dot{x}+\left(\frac{k}{m}\right) x=0
$$

Fonte: Adaptada de (COSSOLINO; PEREIRA, 2010).

Chamando-se:

$$
\omega_{0}=\sqrt{\frac{k}{m}} \quad \zeta=\frac{c}{2 \sqrt{k m}}
$$

Fonte: Adaptada de (COSSOLINO; PEREIRA, 2010).

onde $\omega_{0}$ é a freqüência natural de vibração e $\zeta$ representa a taxa de amortecimento ou apenas amortecimento. Desta forma, pode-se reescrever a equação, utilizando-se os novos parâmetros:

$$
\ddot{x}+2 \zeta \omega_{0} \dot{x}+\omega_{0}^{2} x=0
$$

Fonte: Adaptada de (COSSOLINO; PEREIRA, 2010).

Desta forma assumindo a solução: 


$$
X=\mathrm{e}^{\gamma t}
$$

Fonte: Adaptada de (COSSOLINO; PEREIRA, 2010).

Chega-se a $\gamma$ descrito na forma:

$$
\gamma=\omega_{0}\left(-\zeta \pm \sqrt{\zeta^{2}-1}\right)
$$

Fonte: Adaptada de (COSSOLINO; PEREIRA, 2010).

Assim, a equação acima depende da solução de $\gamma$ :

Para $\zeta>1$ : há duas soluções reais e chamamos de caso superamortecido;

Para $\zeta=1$ : há uma solução real e chamamos de caso criticamente amortecido;

Para $0 \leq \zeta<1$ : há duas soluções complexas e chamamos de caso sub-amortecido.

Os casos superamortecido e criticamente amortecido são considerados não oscilatórios e, portanto, não se enquadram nos parâmetros que cercam o estudo de sinais acústicos, que são fenômenos oscilatórios.

Para a solução $0 \leq \zeta<1$, o sistema sub-amortecido, possui a equação:

$$
\begin{array}{r}
x(t)=A_{0} e^{-\zeta \omega_{0} t} \cos \left(\omega_{d} t+\varphi\right) \\
\text { Fonte: Adaptada de (COSSOLINO; PEREIRA, 2010). }
\end{array}
$$

Onde $A_{0}$ é a amplitude inicial de vibração, $\varphi$ é a fase inicial de vibração e $\omega_{d}$ é chamada de frequência natural amortecida e é descrita por:

$$
\omega_{d}=\omega_{0} \sqrt{1-\zeta^{2}}
$$

Fonte: Adaptada de (COSSOLINO; PEREIRA, 2010).

Que é válido para um modelo conhecido como sistema linear amortecido com um grau de liberdade. Desta forma, assume-se que a ressonância de materiais pode ser vista como uma associação de vários sistemas de um grau de liberdade, e o modelo de vibração é dado por:

$$
x(t)=\left[\sum_{i=1}^{N} A_{i} e^{-\zeta_{i} \omega_{n i} t} \cos \left(\omega_{d i}+\varphi_{i}\right)\right]+R_{w n}
$$


em que $A_{i}, \zeta_{i}, \omega_{\text {in }},{ }_{\text {id }}, \varphi_{i}$, são, respectivamente, amplitude inicial, amortecimento, frequência natural de vibração, frequência natural amortecida e fase inicial do i-ésimo modo de vibração. 0 termo $R_{\omega n}$ é um ruído branco descorrelacionado do sinal.

\subsubsection{Determinação do Amortecimento pelo Método do decremento Logarítmico}

O conceito de amortecimento pode ser estendido para sistemas complexos, embora não exista uma expressão simples para definição de $\zeta$ para sistemas complexos com múltiplos graus de liberdade. $\mathrm{O}$ amortecimento pode ser calculado a partir do comportamento versus tempo de um ou mais pontos do sistema. Para tanto se pode usar o método do decremento logarítmico.

O decremento logarítmico é calculado a partir de um simples impulso provocado no sistema (em vibração livre) através da razão entre duas amplitudes sucessivas do sinal. 0 termo decremento logarítmico refere-se à taxa de redução logarítmica, relacionada com a redução do movimento após o impulso, pois a energia é transferida para outras partes do sistema ou é absorvida pelo próprio elemento.

Este é o método mais utilizado para calcular o amortecimento.

Quando um sistema oscilatório com um grau de liberdade, com amortecimento viscoso é excitado por um impulso, sua resposta vem na forma de decaimento no tempo (Figura 1), dada por:

Figura 1 - Resposta ao Impulso

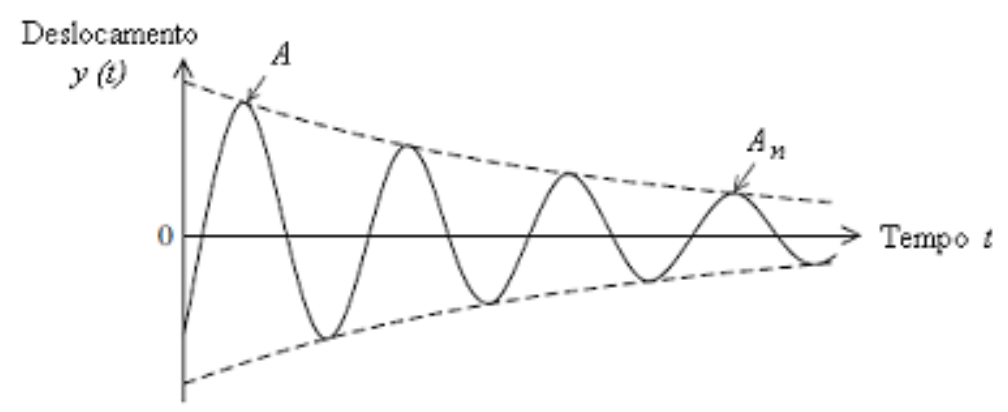

Fonte: (COSSOLINO E PEREIRA, 2010).

$$
\begin{array}{r}
y(t)=y e^{-\varsigma w_{0} t} \operatorname{sen}\left(w_{d} t\right) \\
\text { Fonte: Adaptada de (COSSOLINO; PEREIRA, 2010). }
\end{array}
$$

Esta equação é análoga à equação 8, onde a frequência natural amortecida é dada pela equação 9:

Se a resposta no tempo $t=t_{n}$ é denotada por $y$, e a resposta no tempo $t=t n+2 \pi r / \omega_{d}$ é denotada por $y_{n^{\prime}}$ então, da equação 9 tem-se que:

$$
\frac{y_{n}}{y}=\exp \left(-\zeta \frac{\omega_{0}}{\omega_{d}} 2 \pi r\right), \quad \mathrm{n}=1,2,3, \ldots
$$


Supondo que y corresponda a um ponto no decaimento da função com amplitude igual a $A$, e que $y_{n}$ corresponde ao pico, $r$ ciclos mais tarde, com magnitude $A_{n}$. Assim, tem-se que:

$$
\frac{A_{n}}{A}=\exp \left(-\zeta \frac{\omega_{0}}{\omega_{d}} 2 \pi r\right)=\exp \left[-\frac{\zeta}{\sqrt{1-\zeta^{2}}} 2 \pi r\right]
$$

Fonte: Adaptada de (COSSOLINO; PEREIRA, 2010).

Através da análise de um modelo viscoelástico com um grau de liberdade e utilizando-se o valor da frequência natural amortecida (equação 9 ), chega-se á fórmula do decremento logarítmico $(\delta)$ :

$$
\delta=\frac{1}{n} \ln \left(\frac{A_{1}}{A_{n}}\right)=\frac{2 \pi \zeta}{\sqrt{1-\zeta^{2}}}
$$

Fonte: Adaptada de (COSSOLINO; PEREIRA, 2010).

Para o amortecimento $(\zeta)$, tem-se que:

$$
\zeta=\frac{1}{\sqrt{1+(2 \pi / \delta)^{2}}}
$$

Fonte: Adaptada de (COSSOLINO; PEREIRA, 2010).

Para casos em que o amortecimento é muito pequeno $(\delta<0,1)$, a frequência natural amortecida é praticamente igual frequência natural, ou seja, $\omega_{d}=\omega_{0}$, e então a equação 13 pode ser escrita como:

$$
\frac{A_{n}}{A} \cong \exp (-\zeta 2 \pi r)
$$

Fonte: Adaptada de (COSSOLINO; PEREIRA, 2010).

Com isso define-se $\zeta$ :

$$
\zeta=\frac{1}{2 \pi} \cdot \frac{1}{n} \ln \left(\frac{A_{1}}{A_{n}}\right)=\frac{\delta}{2 \pi} \quad \text { para } \zeta<0,1
$$

Fonte: Adaptada de (COSSOLINO; PEREIRA, 2010).

Após n períodos, o amortecimento pode ser calculado em função da razão de outra amplitude.

A expressão acima foi usada para análise de sinais para determinação do amortecimento dos sinais captados de cordas conforme apresentado no capitulo três. 


\subsection{Objetivo}

O objetivo deste artigo é um estudo de características relevantes à resposta dada pelo instrumento quando este é submetido a um impulso. Tal pesquisa objetiva caracterizar qualidades com relação ao amortecimento dos sinais gerados por violões de fabricação artesanal e comercial.

\section{METODOLOGIA}

Os violões utilizados neste trabalho são da marca Gianini e Takamine de fabricação industrial e de qualidades subjetivas conhecidas no meio musical, além de outros três da marca Barros de fabricação artesanal fornecido e por um luthier experiente, colaborador deste projeto que se acredita subjetivamente ser de qualidades superiores.

O Luthier propõe para este estudo a seguinte classificação de qualidade em ordem decrescente dos instrumentos utilizados:

Tipo A: Barros 6 cordas_abeto_hab1_2012 corda_gianini_ginga

Tipo B: Barros 7 cordas_cedro_2012_corda_gianini_ginga

Tipo C: Barros_6cordas_hab2_cedro_2012_corda_gianini_ginga

Tipo D: Takamine_corda_gianini_ginga

Tipo E: Gianini_7cordas_corda_gianini_ginga

A comparação é feita através do amortecimento da frequência fundamental de várias notas musicais gravadas dos violões utilizados seguindo os procedimentos dos tópicos 2.1 a 2.5. Para todos os instrumentos foi utilizado o mesmo jogo de cordas, da marca gianini ginga.

\subsection{Captação dos Sinais}

Os sinais a serem utilizados neste trabalho serão captados a partir da gravação de respostas sonoras nos tampos dos violões mencionados anteriormente. Tais sinais são, na realidade, reproduções da escala musical temperada com as devidas frequências fundamentais e seus harmônicos. Essas respostas sonoras são gravadas em arquivos digitais, inicialmente no formato wav, usando um software chamado cool edit 2000. Em seguida, os sinais são transferidos para o software matlab (R2010a), onde podem ser lidos para posteriormente serem trabalhados no domínio do tempo e frequência.

\subsection{Conversão de Sinais para o Domínio da Frequência}

Os sinais captados pelo processo de gravação, são convertidos em frequências através da FFT (Fast Fourier Transform) que estabelece a relação de transferência de domínio do tempo e frequência para posterior análise espectral.

Através do domínio da frequência pode-se analisar quais frequências são excitadas, podendo assim, estabelecer a freqüência fundamental da nota musical e os seus harmônicos. 


\subsection{Filtragem de Sinais}

Após feita a separação da frequência fundamental e seus harmônicos, com o auxilio do Matlab, aplica-se um filtro passa banda que consiste em delimitar uma região de frequências, neste caso, em torno da frequência fundamental. Onde os limites são a frequências de corte inferior e superior que estão localizadas na base da frequência excitada. Essa filtragem gera um novo sinal, no domínio do tempo, representado apenas por uma das frequências excitadas.

\subsection{Teste de filtragem do sinal}

Com o sinal filtrado é necessário retorná-lo para o domínio da frequência para verificar se realmente o filtro funcionou. Para tal, aplica-se novamente a FFT. deverá aparecer somente um pico mais evidente na faixa de frequência selecionada.

\subsection{Análise do amortecimento}

Com o sinal filtrado e verificado na frequência estabelecida é necessário retornar ao domínio do tempo para obter os valores de amplitudes usados no cálculo de amortecimento. Isso é feito através da transformada inversa de Fourier (IFFT).

Os valores das taxas de amortecimento (equação 17) são obtidos através do método do decremento logarítmico (equação 14) que é obtido através da razão entre valores de duas amplitudes sucessivas do sinal. Neste trabalho são usados os valores de amplitude do primeiro e décimo ciclo.

\section{RESULTADOS}

Todos os sinais usados neste trabalho foram submetidos aos procedimentos descritos no capítulo dois, com o objetivo de gerar gráficos no espaço amplitude versus tempo, obter as amplitudes de primeiro e décimo ciclo para posterior cálculo do amortecimento, Como demonstrado na figura 2. Para o exemplo em questão ( $A_{4}$ filtrado num passa banda de $\left.440 \mathrm{Hertz}\right)$ os cálculos são os seguintes:

$$
\begin{array}{r}
\delta=\frac{1}{n} \ln \left(\frac{A_{1}}{A_{n}}\right)=\frac{2 \pi \zeta}{\sqrt{1-\zeta^{2}}}=\frac{1}{10} \ln \left(\frac{0,4285}{0,3984}\right)=0,007283421 \\
\text { Fonte: Adaptada de (COSSOLINO; PEREIRA, 2010). }
\end{array}
$$

Em termos de amortecimento $(\zeta)$, tem-se:

$$
\begin{array}{r}
\zeta=\frac{1}{2 \pi} \cdot \frac{1}{n} \ln \left(\frac{A_{1}}{A_{n}}\right)=\frac{\delta}{2 \pi}=\frac{0,007283421}{2 \cdot \pi}=0,001159192 \\
\text { Fonte: Adaptada de (COSSOLINO; PEREIRA, 2010). }
\end{array}
$$

Todo o procedimento descrito é repetido para notas musicais da escala temperada de $\mathrm{Mi}$ da segunda oitava $\left(E_{2}, 82,43\right.$ hertz) até Fá sustenido da quinta oitava $\left(F_{5} \# ; 739,99\right.$ hertz) para os cinco violões pesquisados. 
$\mathrm{Na}$ tabela 1 estão todos os valores calculados das taxas de amortecimento. A primeira coluna representa a nota musical com a referência no sistema americano [Dó (C), Ré (D), Mi (E), Fá (F), Sol (G), Lá (A), Si (B)]. Na segunda coluna estão as frequências fundamentais encontradas para cada nota musical e da terceira até a sétima coluna estão os valores calculados do amortecimento das várias notas musicais em cada instrumento analisado.

Figura 2 - Sinal filtrado no domínio do tempo com as amplitudes $A_{1}$ e $A_{10}$ da nota Lá da quarta oitava ( $A 4$, frequência fundamental $=440$ hertz) de um violão da marca Takamine.

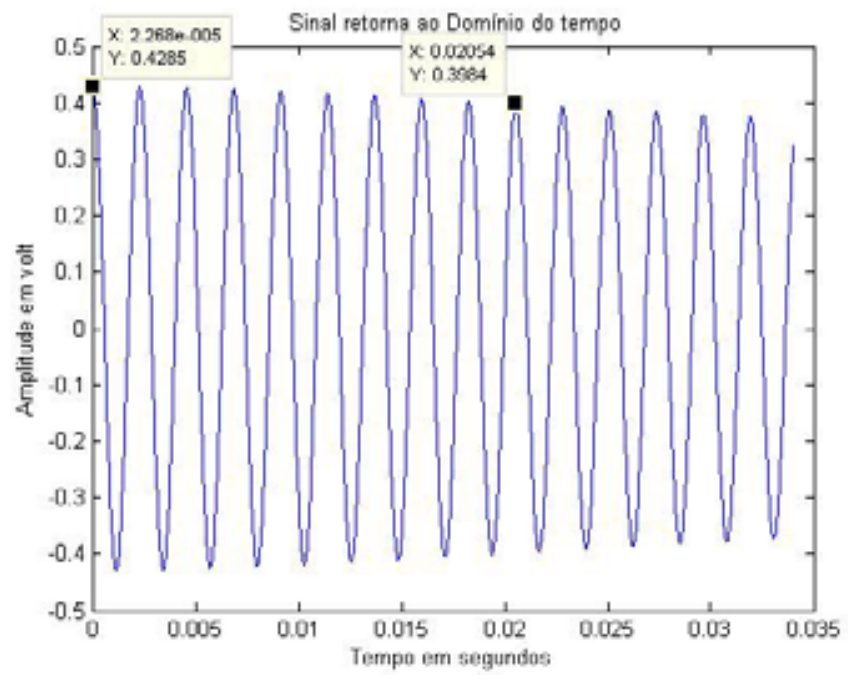

Fonte: ( DOS AUTORES, 2014). 
Tabela 1 - Taxas de amortecimento dos cinco violões (E2 a F5\#).

Planilha de dados comparando o amortecimento de cinco violões

\begin{tabular}{|c|c|c|c|c|c|c|}
\hline \multirow[b]{2}{*}{$\begin{array}{c}\text { Nota } \\
\text { musical }\end{array}$} & \multirow[b]{2}{*}{$\begin{array}{c}\text { Frequência } \\
\text { Fundamental } \\
\text { (Hertz) }\end{array}$} & \multicolumn{5}{|c|}{ Amortecimento (adimensional) } \\
\hline & & $\begin{array}{l}\text { Barros } \\
\text { Tipo A }\end{array}$ & $\begin{array}{l}\text { Barros } \\
\text { Tipo B }\end{array}$ & $\begin{array}{l}\text { Barros } \\
\text { Tipo C }\end{array}$ & $\begin{array}{c}\text { Takamine } \\
\text { Tipo D }\end{array}$ & $\begin{array}{l}\text { Gianini } \\
\text { Tipo E }\end{array}$ \\
\hline E2 & 82,43 & $1,17818130 \mathrm{E}-02$ & 0,01164831 & 1,177910E-02 & $1,202 \mathrm{E}-02$ & $1,193 \mathrm{E}-02$ \\
\hline $\mathrm{F} 2$ & 87,31 & $8,25633725 \mathrm{E}-03$ & $8,5301 \mathrm{E}-03$ & $7,148158 \mathrm{E}-03$ & 8,338E-03 & $8,682 E-03$ \\
\hline F2\# & 92,5 & $9,66130261 \mathrm{E}-03$ & 1,6161E-03 & 1,396792E-03 & 1,617E-03 & $1,738 \mathrm{E}-03$ \\
\hline $\mathrm{G} 2$ & 98 & $1,77123807 \mathrm{E}-03$ & $4,2830 \mathrm{E}-03$ & 4,233073E-03 & $4,180 \mathrm{E}-03$ & $4,370 \mathrm{E}-03$ \\
\hline G2\# & 103,83 & $5,59841003 E-04$ & $3,8862 \mathrm{E}-04$ & $7,708461 \mathrm{E}-04$ & $3,147 \mathrm{E}-03$ & 7,376E-04 \\
\hline A2 & 110 & 4,95493981E-04 & 1,0394E-03 & $3,662310 \mathrm{E}-04$ & $1,698 \mathrm{E}-03$ & $2,288 \mathrm{E}-04$ \\
\hline A2\# & 116,54 & $2,51173647 \mathrm{E}-04$ & $5,5267 \mathrm{E}-04$ & 2,096192E-04 & $4,353 E-04$ & $2,024 \mathrm{E}-04$ \\
\hline B2 & 123,47 & $5,14315080 \mathrm{E}-04$ & $1,6804 \mathrm{E}-04$ & $3,781001 E-04$ & $5,453 E-04$ & $3,957 \mathrm{E}-04$ \\
\hline C3 & 130,81 & $4,18875350 \mathrm{E}-04$ & $1,6520 \mathrm{E}-04$ & $3,814492 \mathrm{E}-04$ & 2,807E-04 & $2,473 \mathrm{E}-04$ \\
\hline C3\# & 138,59 & $2,72525653 \mathrm{E}-04$ & $1,0236 \mathrm{E}-04$ & $3,070239 E-04$ & $2,040 \mathrm{E}-04$ & $1,214 \mathrm{E}-04$ \\
\hline D3 & 146,83 & $9,29522452 E-04$ & $5,1965 \mathrm{E}-04$ & 8,783809E-04 & $9,406 \mathrm{E}-04$ & $5,602 \mathrm{E}-04$ \\
\hline D3\# & 155,56 & $6,11242405 E-04$ & $2,4675 \mathrm{E}-04$ & $3,482330 \mathrm{E}-04$ & $4,945 \mathrm{E}-04$ & $5,035 \mathrm{E}-04$ \\
\hline E3 & 164,81 & $9,98903026 \mathrm{E}-04$ & 1,3169E-04 & $2,312802 E-04$ & $4,525 E-04$ & $1,162 \mathrm{E}-04$ \\
\hline F3 & 174,61 & $1,34049264 \mathrm{E}-03$ & $3,2170 \mathrm{E}-04$ & $5,713298 E-04$ & $5,205 E-04$ & 2,864E-04 \\
\hline F3\# & 185 & $2,32658285 E-03$ & $3,6705 \mathrm{E}-04$ & 1,394953E-03 & $9,707 \mathrm{E}-04$ & $5,473 \mathrm{E}-04$ \\
\hline G3 & 196 & $1,08834402 \mathrm{E}-03$ & $7,7532 \mathrm{E}-04$ & 3,331207E-03 & 1,392E-03 & $5,079 \mathrm{E}-04$ \\
\hline G3\# & 207,65 & $3,38212373 \mathrm{E}-04$ & $7,4221 \mathrm{E}-04$ & 1,858666E-03 & $1,355 \mathrm{E}-03$ & $1,938 \mathrm{E}-04$ \\
\hline A3 & 220 & $4,02096624 \mathrm{E}-04$ & 1,876E-03 & $7,185386 \mathrm{E}-04$ & $9,078 \mathrm{E}-04$ & $3,010 \mathrm{E}-03$ \\
\hline A3\# & 233,08 & $4,70536672 \mathrm{E}-04$ & $2,0729 \mathrm{E}-03$ & 7,220990E-04 & $7,142 \mathrm{E}-04$ & $2,487 \mathrm{E}-03$ \\
\hline B3 & 246,94 & $2,84519124 \mathrm{E}-04$ & 2,7667E-03 & $2,413983 E-04$ & $2,161 \mathrm{E}-04$ & $2,444 \mathrm{E}-04$ \\
\hline C4 & 261,63 & 9,26349061E-04 & 1,3289E-03 & $9,414609 E-04$ & $1,383 E-03$ & $7,785 \mathrm{E}-04$ \\
\hline C4\# & 277,18 & $4,70818010 \mathrm{E}-04$ & $7,3715 \mathrm{E}-04$ & 4,897412E-04 & $7,619 \mathrm{E}-04$ & 8,019E-04 \\
\hline D4 & 293,66 & $3,58948057 \mathrm{E}-04$ & $3,1185 \mathrm{E}-04$ & 4,103617E-04 & $2,674 \mathrm{E}-04$ & $1,180 \mathrm{E}-04$ \\
\hline D4\# & 311,13 & $3,45024108 \mathrm{E}-04$ & 4,8790E-04 & 5,244069E-04 & $3,823 \mathrm{E}-04$ & $4,361 \mathrm{E}-04$ \\
\hline E4 & 329,63 & $5,34384554 \mathrm{E}-04$ & $2,6224 \mathrm{E}-04$ & $3,719387 \mathrm{E}-04$ & $6,906 \mathrm{E}-04$ & 4,078E-04 \\
\hline F4 & 349,23 & $1,75340955 \mathrm{E}-04$ & $8,0119 \mathrm{E}-06$ & 3,613938E-04 & 2,073E-04 & $1,263 \mathrm{E}-04$ \\
\hline F4\# & 369,99 & $4,41708771 \mathrm{E}-04$ & $4,6918 \mathrm{E}-04$ & $5,174644 \mathrm{E}-04$ & $6,090 \mathrm{E}-04$ & $2,655 \mathrm{E}-04$ \\
\hline G4 & 391,99 & $5,46691752 \mathrm{E}-04$ & $3,7039 \mathrm{E}-04$ & $2,673601 \mathrm{E}-04$ & $4,211 \mathrm{E}-04$ & 4,906E-04 \\
\hline G4\# & 415,31 & $7,12402022 \mathrm{E}-04$ & 6,4487E-04 & 2,842466E-04 & 6,707E-04 & 7,366E-04 \\
\hline A4 & 440 & $2,03553949 E-04$ & $2,1583 \mathrm{E}-04$ & 2,159974E-04 & $2,243 E-04$ & $1,608 \mathrm{E}-04$ \\
\hline A4\# & 466,16 & $2,41881608 \mathrm{E}-04$ & $5,0118 \mathrm{E}-04$ & $2,076406 \mathrm{E}-04$ & $3,916 \mathrm{E}-04$ & 1,707E-04 \\
\hline B4 & 493,88 & $3,38862850 \mathrm{E}-04$ & 2,9334E-04 & $4,361314 \mathrm{E}-04$ & $1,873 E-04$ & $1,362 \mathrm{E}-04$ \\
\hline C5 & 523,25 & $5,51767787 \mathrm{E}-04$ & $2,8896 \mathrm{E}-04$ & $1,540710 \mathrm{E}-04$ & $2,902 \mathrm{E}-04$ & $2,163 \mathrm{E}-04$ \\
\hline C5\# & 554,36 & $1,50223440 \mathrm{E}-04$ & $2,1155 \mathrm{E}-04$ & 2,888432E-04 & $3,415 \mathrm{E}-04$ & $2,815 \mathrm{E}-04$ \\
\hline D5 & 587,32 & $2,57663169 \mathrm{E}-04$ & $1,4935 \mathrm{E}-04$ & $2,358145 \mathrm{E}-04$ & $3,278 \mathrm{E}-04$ & $7,856 \mathrm{E}-05$ \\
\hline D5\# & 622,24 & $1,10708901 \mathrm{E}-04$ & $1,2648 \mathrm{E}-04$ & 1,439815E-04 & $2,110 \mathrm{E}-05$ & $1,420 \mathrm{E}-04$ \\
\hline E5 & 659,24 & $4,52373885 \mathrm{E}-04$ & $1,2744 \mathrm{E}-04$ & $4,021748 \mathrm{E}-04$ & $1,250 \mathrm{E}-04$ & $3,389 \mathrm{E}-04$ \\
\hline F5 & 698,46 & $5,90546019 \mathrm{E}-04$ & $3,4054 \mathrm{E}-04$ & $2,643330 \mathrm{E}-04$ & $3,383 E-04$ & $5,340 \mathrm{E}-04$ \\
\hline F5\# & 739,99 & $2,04525685 \mathrm{E}-04$ & $5,9954 \mathrm{E}-04$ & $1,904755 \mathrm{E}-04$ & $2,353 E-04$ & $2,012 \mathrm{E}-04$ \\
\hline
\end{tabular}

Fonte: ( DOS AUTORES, 2014). 
Os resultados para cada violão estão nas figuras $3(a, b, c, d, e)$ que mostram no geral taxas de amortecimento com valores muito parecidos para os cinco instrumentos utilizados nesta análise, porém se percebe que as notas com frequências abaixo de $100 \mathrm{~Hz}$ têm valores mais altos do que as demais. Destacam-se também com relação aos valores de amortecimentos, as notas de frequências entre 150 e $300 \mathrm{~Hz}$, sendo que as demais, de 300 a $750 \mathrm{~Hz}$, apresentam valores menores e muito parecidos com variação entre 0 e 0,001 .

Figura 3 - Amortecimento versus Frequência nos cinco violões

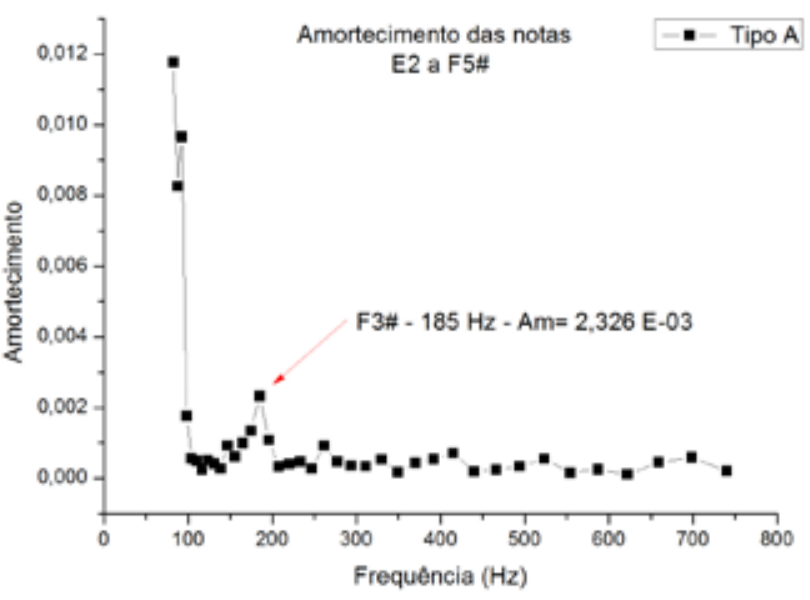

(a) - Tipo A (Barros)

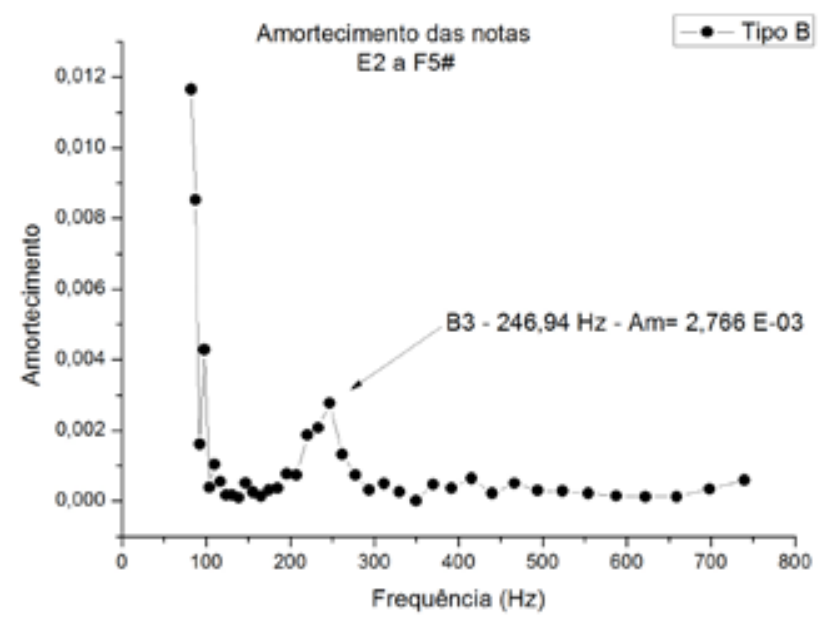

(b) - Tipo B (Barros)

Fonte: ( DOS AUTORES, 2014). 


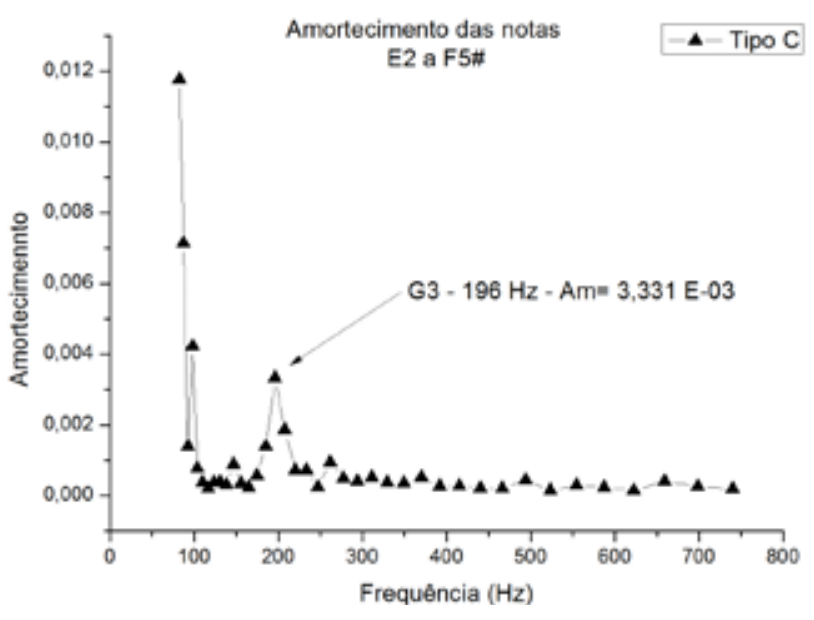

(c) - Tipo C (Barros)

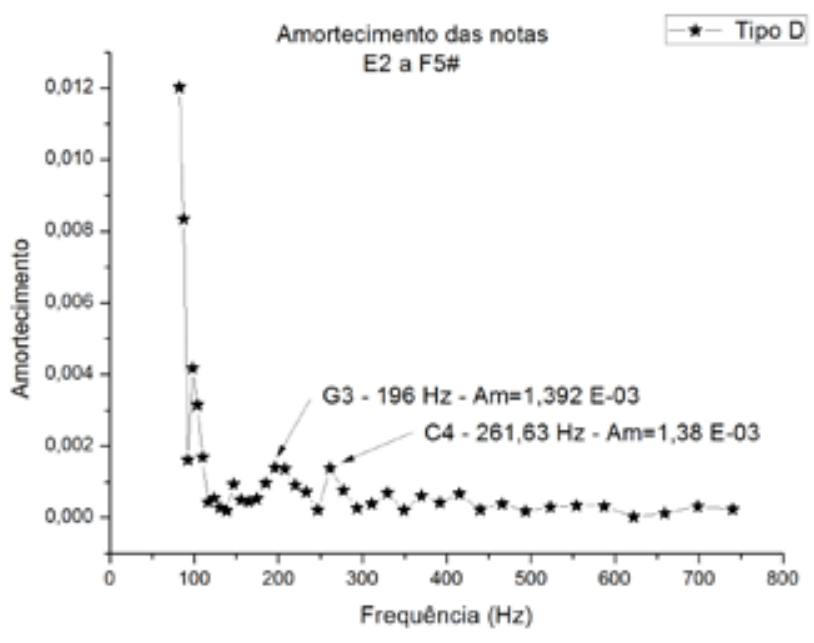

(d) - Tipo D (Takamine)

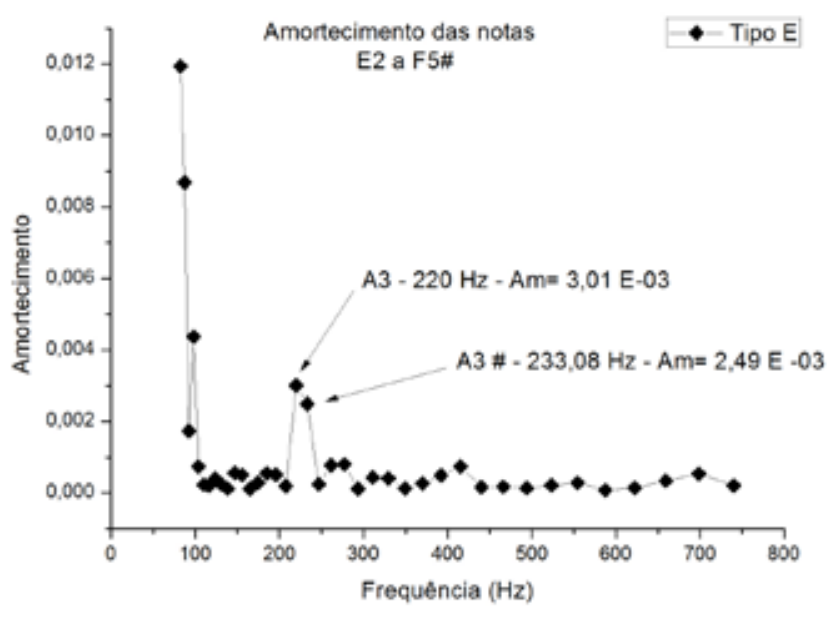

(e) - Tipo E (Gianini)

Fonte: ( DOS AUTORES, 2014). 
As frequências abaixo de $100 \mathrm{~Hz}$ (baixa frequência) apresentam valores elevados de amortecimento com relação às demais (variação entre 0 e 0,012 ), mas muito parecidos em todos os instrumentos. Porém, nas notas D3\# a D4 com frequências de $155,56 \mathrm{~Hz}$ a $293,66 \mathrm{~Hz}$ é possível estabelecer algumas comparações.

Os três instrumentos artesanais Barros (tipo A, B e C) apresentam taxas de amortecimento significativas em relação aos demais para algumas notas.

Considerando uma faixa média de valores como valor mínimo $(0,0015)$, o violão $A$ apresenta uma nota acima ( $F 3 \#)$, o violão $B$ três notas ( $A 3, A 3 \#$ e B3), o violão $C$ duas notas ( $G 3$ e $G 3 \#)$, o Takamine (D) não apresenta nenhuma taxa acima deste valor, porém o violão Gianini $(E)$, se destaca com valores significativos nas notas A3 e A3\#.

As notas D4\# a F5\# com frequências entre 311,13 a 739,99 hertz apresentam taxas de amortecimento com valores menores, abaixo de 0,0008 , não permitindo uma distinção muito clara entre os instrumentos.

\section{CONCLUSÕES}

A avaliação dos resultados mostra que a distribuição das taxas de amortecimento com relação às frequências fundamentais, que armazenam a maior parcela da energia de cada sinal gravado, é muita parecida para os cinco instrumentos analisados. Porém, há que se destacar que as notas com frequências de 150 a 300 Hertz apresentam resultados mais significativos indicando certa comparação entre os instrumentos.

Os violões tipo A, B e C e E apresentam valores acima da faixa média considerada $(0,0015)$, diferentemente do violão $D$. As maiores taxas são em primeiro do violão $C$ (nota $G 3$ ), em segundo, violão $E$ (nota $A 3$ ), em terceiro, violão B (nota B3), em quarto, tipo $A$ (nota F3\#) e em quinto, tipo D (nota G3). No geral, para esta faixa, Takamine apresenta as menores taxas.

Numa análise geral, não dá para estabelecer uma ordem de classificação com relação ao amortecimento da nota fundamental, pois estas variam muito a cada nota. Mas por outro lado, o cálculo do amortecimento do sinal é uma importante ferramenta para o estudo de sinais, pois representa a taxa de dissipação de energia no meio material.

Pode-se perceber que os detalhes construtivos ou os tipos de madeira utilizados tanto no processo artesanal dos violões Barros ou no processo mais industrializado dos violões Gianini e Takamine podem influenciar significativamente na qualidade do sinal gerado, ou neste caso, no amortecimento dos mesmos. Mas é necessário um estudo mais aprofundado de outros parâmetros para entender melhor tais diferenças.

\section{REFERÊNCIAS}

COSSOLINO L.C.; PEREIRA A.H.A. - "Amortecimento: classificação e métodos de determinação" Universidade de São Carlos, 2010.

JANSSON, ERIK V. - "Acoustics for the Guitar Player" - seminar organized by the Committee for the Acoustics of Music, Publications issued by the Royal Swedish Academy of Music No. 38, 1983

WRIGHT, H. - "The Acoustics and Psychoacoustics of the Guitar" - Department of Physics and Astronomy, University of Wales, 1996. 